\title{
Working Towards Strong Wireless Group Communications: The Janus Architecture
}

\author{
J. S. Pascoe $\uparrow \ddagger$ V. S. Sunderam $\ddagger$ and R. J. Loader $\dagger \neq$ \\ $\dagger$ Department of Computer Science \\ The University of Reading \\ United Kingdom \\ RG6 6AY \\ J.S.Pascoe@reading.ac.uk \\ Roger.Loader@reading.ac.uk \\ ¥Math \& Computer Science \\ Emory University \\ Atlanta, Georgia \\ 30322 \\ vss@mathcs.emory.edu
}

\begin{abstract}
Strong wired group communication systems have been employed in numerous applications, often characterised by some degree of replication. With the development of wireless technologies, a new series of group applications is emerging and due to the effectively asymmetric characteristics of these networks, existing group communication systems can not be employed directly. Differences between wireless and wired networks in terms of connectivity, latency, bandwidth and other characteristics necessitate alternative algorithms and mechanisms, especially in group communication protocols. In particular, protocols must adapt to highly variable network characteristics in the wireless domain, whilst providing standard group communication semantics and delivering traditional qualities of service. In addition, arbitration between wired and wireless communication entities is required to reconcile the order-of-magnitude (or more) difference in network parameters between the two.

The Janus project is developing a strong group communications framework for use in wireless environments. It is envisaged that the findings from our research, as well as the software that results, will be of value in a number of emerging domains such as collaborative computing and multiway application sharing that are moving from fully wired to hybrid wireless and land-line networks.
\end{abstract}

Keywords - Janus, group communication, wireless networking, preemptive adaptation, trouble spots, failure resilience.

\section{Introduction}

The use of mobile devices such as network-enabled PDA's and notebooks is rapidly growing and becoming a mainstream mode of portable computing and communications. Although some platforms are proprietary (e.g. Palm VIIx), current trends are towards wireless infrastructures supporting IP, facilitating widespread deployment of traditional applications. For example, notebook computers on 802.11 networks are commonplace within buildings, and wireless infrastructures supporting PDA's and hand-helds are becoming increasingly prevalent in metropolitan areas. In these usage scenarios, standard (wired network based) applications execute without the need for modifications to the application or the protocol. Currently, typical applications are mail, web browsing, telnet etc., that are not substantively affected by the latency, bandwidth, and connectivity problems that characterize wireless networks. In other words, the pairwise and clientserver nature of common applications can tolerate the lowered quality found in wireless 
networks e.g. a user experiencing lack of coverage while moving in a hallway simply reloads a web page as soon as coverage is re-established, very much like reloading a stalled page on a wired workstation.

However, a number of other current and emerging applications are much more sensitive to the inherently low bandwidth, high latency, and most of all, intermittent connectivity often encountered in wireless networks. This is especially true of applications based on strong group protocols, such as collaborative data and document sharing, server-less chat, application multiplexing, and multiway audio. In multi-party protocols, group membership operations are highly sensitive to actual or perceived joining and leaving by members. Mobile devices encountering areas of disconnection can cause very expensive group operations to be erroneously triggered. Similarly, in several multicast and group communication modes, marked disparities in delivery latencies or bandwidths between different group members can result in severe protocol degradation. For example, a series of reliable multicasts can result in buffer overflows at land-based nodes due to long acknowledgment delays from even a single wireless node. At least in the near future, this large difference in quality between wired and wireless networks is expected to prevail. Therefore, new approaches to providing protocol support for multiway applications in hybrid networks is of crucial importance.

This paper is based on the premise that transport quality in wireless networks is dependent on spatial and environmental factors, i.e. regions within the coverage area that are susceptible to network degradation either due to their location, or due to disturbances (thunderstorms or radiation from a CRT monitor). However, knowledge of these trouble spots (or the assumption of their existence) can be used by protocols to preemptively adapt, correct, or otherwise account for the lowered quality when a mobile device encounters such an area. Knowledge of trouble spots is based on both static information gained from prior visits to the area as well as by real-time monitoring of the network from within each mobile device. Adaptation can take many forms, ranging from increased buffering to dynamically changing the QoS of a group communication channel. This project is developing methodologies and protocols, that will support adaptive group communications in hybrid wireless and wired networks.

\section{Background}

A strong model of group communication differs from weak counterparts by including a formalized notion of membership, either through an explicit group membership service or as an element of the distributed state. An example of a strong group communication system is ISIS [3] whereas an example of a weak model is IP-multicast [5]. ISIS was a general group communications platform, first proposed by Birman, that introduced the notion of Virtual Synchrony [4] and in doing so was successfully employed in over 300 projects. The original virtual synchrony assumed a primary partition model and as such, was vulnerable to problems arising through network partitions. This issue inspired several new projects which aimed to address partitionable group communications; two of the more notable being Totem [11, 1, 10] and Transis [6]. The Totem group developed a new model of virtual synchrony, termed, Extended Virtual Synchrony which essentially permitted processes to operate in minority partitions, providing that for a given partition, consensus could be formed on its membership. The Transis system, due to Dolev and Malki, provided a large scale hierarchical multicast service that was tolerant of network partitions and leveraged IP-multicast. Transis contributed extensively to group 
communications theory, it introduced a number of significant results and later inspired parts of Horus [15], the successor to ISIS. The Horus framework is based around the notion of protocol composition, that is, Horus provides a catalog of components that can be quickly composed to provide tailored group communications solutions. This 'building block' approach not only provides genuinely re-usable solutions to group applications, but also supports additional project phases e.g. formal verification. Current projects such as InterGroup [2], Spinglass and Ensemble [14] are exploring the issues of scalability and security in group communications.

\section{Architecture}

The envisaged model consists of two sub-groups of communicating entities: $M$ entities on wired hosts (that form a group in the traditional sense) and $N$ entities in the wireless domain. Periodically, both wired and wireless hosts may join and leave a session or heavyweight group, and within a session, they may join and leave lightweight communication channels that support different levels of Quality of Service. Pragmatically, this interaction is facilitated through Janus ${ }^{1}$ like proxies, that arbitrate between the asymmetric characteristics of the two networks. Thus, a Janus group consists of $M$ wired hosts, $N$ wireless hosts and $P$ proxies.

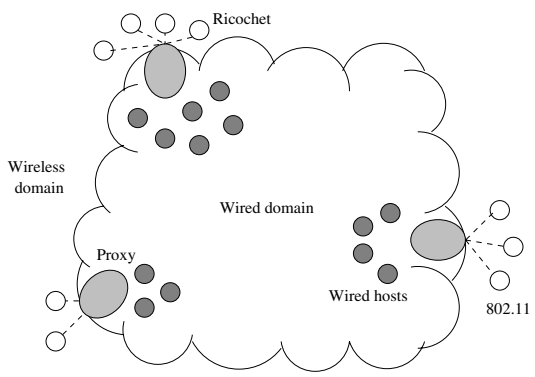

Fig. 1. The envisaged architecture.

The prototype implementation of Janus takes the form of a UDP-based user space protocol. Subsequent projects will then be able to leverage Janus through an API, that is, applications simply link against the Janus libraries and avail the services. In designing Janus, we target three levels of device (PDA, laptop and stationary workstation), just as we target two types of network (802.11 and metropolitan infrastructures). The prototypical work is Linux based and in order to use the system, Janus proxies (configured for the required application) are instantiated on fixed machines in the wired domain. Each wireless device then connects to a proxy and in doing so empowers the proxy to transfer messages, handle synchronization and perform any other function required in the wired domain on its behalf. In sections 4 and 5 we discuss the major challenges that the project is striving to overcome and present an outline of the projects technical direction.

\footnotetext{
${ }^{1}$ Derived from the two-headed Roman god of civil order.
} 


\section{Challenges}

Preliminary experimentation into the Janus concept has shown that the proposed work is feasible and should contribute a novel framework that will have application in several areas of research. In the process, our experiments have highlighted a number of research issues which are central to the projects foundation. Thus, the fundamental areas of research are described below. It is noteworthy to add, that the maintenance of the virtual synchrony semantics is considered paramount in Janus. The virtually synchronous model of group communication has been widely adopted, and as such, it is critical that Janus does not infringe or invalidate this notion both in terms of retrofitting the framework and also leveraging prior research in new projects.

\subsection{Wired and Wireless Network Arbitration}

One of the key questions being addressed by the project is the notion of arbitration between the wired and wireless domains. Wired and wireless networks roughly exhibit asymmetric characteristics, that is, hosts connected to a wired network can exchange messages through low latency, high bandwidth channels, whereas, wireless networks exhibit a much higher degree of latency and currently provide only a fractional proportion of the bandwidth. Also, it is not possible to make assumptions related to the availability of a wireless host. Experience has shown that wireless networks are riddled with small pockets of non-communication, that is, as a mobile user moves throughout the network, a certain level of intermittent connectivity may be experienced. Preliminary experimentation has largely attributed this effect to geographic phenomenon [8, 12], however, other more dynamic factors (such as the weather) can also cause mobile devices to become arbitrarily uncontactable for a priori unknown periods of time. In contrast, hosts connected to a wired network are often highly available with loss of contact being considered an immediate indication of failure.

Given these differences, we postulate that in order to span the two domains, it is necessary to introduce an arbitration entity. This proxy will mediate the transfer of messages between the two environments, providing a highly available participant in the wired domain whilst sympathetically adapting to the intermittent connectivity of the wireless device. Note that this differs from the traditional role of a proxy. In other scenarios, proxies are employed as buffers or caches to increase the perceived availability of a resource (e.g. web caching). In Janus, the notion of a proxy is more complex i.e. a Janus proxy will synchronize, co-ordinate and perform required actions in the wired domain for $n$ wireless hosts simultaneously.

\begin{tabular}{||c|c|c|c|c|c|c||}
\hline & Wired -1 & Wired $-\mathrm{r}$ & $802.11-\mathrm{s}$ & $802.11-\mathrm{m}$ & Metro. $-\mathrm{s}$ & Metro. $-\mathrm{m}$ \\
\hline PL (\%) & 0 & 0.2 & 0 & 1 & 0 & 0.4 \\
L (ms) & 0.2 & 71.4 & 1.724 & 2.721 & 189.3 & 241.4 \\
BW (KB/s) & 7773 & 48.1 & 637 & 524 & 16.4 & 13.5 \\
\hline
\end{tabular}

Table 1. Quantitative differences between wired and wireless networks. The packet loss, latency and bandwidth measurements are the average results from sending 1000 ICMP packets across the Internet. The abbreviations $l, r, s$ and $m$ equate respectively to local, remote, stationary and moving. Note that the metropolitan area wireless network measurements were made using the Ricochet infrastructure in clear reception areas. 


\begin{tabular}{||c|c|c|c||}
\hline & PDA & Laptop & Desktop \\
\hline Mobility & very high & less than PDA & not mobile \\
Network & wireless & wireless \& wired & wired \\
Memory size & $\approx 32 \mathrm{Mb}$ & $32 \mathrm{Mb}-256 \mathrm{Mb}$ & $\approx 1 \mathrm{~Gb}$ \\
Processor & $\approx 233 \mathrm{Mhz}$ & $\approx 750 \mathrm{Mhz}$ & $\approx 1.5 \mathrm{Ghz}$ \\
Storage & minimal & abundant & abundant \\
Battery issues & $\checkmark$ & $\checkmark$ & $\times$ \\
\hline
\end{tabular}

Table 2. Typical characteristics of devices in a Janus group.

Janus is envisioned for use in group applications where a range of mobile devices are required to interface to a network of stationary wired hosts. With the advent of metropolitan area wireless networks and sophisticated mobile computers, the notion of a ubiquitously connected mobile device is becoming more realistic. However, we postulate that mobile computing and communications capabilities will continue to be somewhat different from (lower than) land based services for the near-to-medium term (see tab. 2). This imposes a number of constraints on the Janus client interface. Due to limitations of processing speed, memory and storage, it is not feasible to execute large group communication applications (intended for desktop use) on small portable devices. Indeed, experimentation has shown that a commercial off the shelf Personal Data Assistant can comfortably support a graphical user interface plus minimal amounts of processing. However, such devices are not capable of running large environments or physical simulations. Note, that this is not a general observation; for more powerful mobile computers (e.g. laptops), it is beneficial to utilize the local resources where possible. For this reason, a later project phase will enhance the client component of the Janus framework to support an element of adaptability, migrating processing load where necessary but harnessing local resources where available. This will provide a level of 'future-proofing' as mobile devices develop.

\subsection{Fault-tolerance}

Traditional fault-tolerance in group communication systems has been based around the reasoned assumption of a fail-stop model of failure, that is, once a host has suffered a failure, it does not participate in the group any longer (although it may rejoin as a new member). In a wired environment, this is a justified supposition since the majority of failures are due to host crashes or network partitioning. However, in a wireless environment, mobile hosts are susceptible to intermittent connectivity and so a more insightful model of failure is required.

As noted above, our preliminary experimentation with metropolitan area wireless networks revealed that there are pockets of non communication scattered throughout the network. Trouble spots can be categorized as either static (which are typically due to geographic phenomenon) or dynamic (intermittent / atmospherically related). In either case, the user can experience temporary disconnects (lasting for arbitrary lengths of time) and message garbling (due to problems such as echoing and high path loss [13, 7,9]). Traditional failure detectors have been based on heartbeat mechanisms viz. each host periodically probes the liveness of the other hosts in its view. Should a heartbeat message remain unacknowledged for a threshold period of time, then this indicates a failure and a membership algorithm is invoked to restore the health of the session. When tested, the use of a heartbeat mechanism in the wireless domain results in a large number 


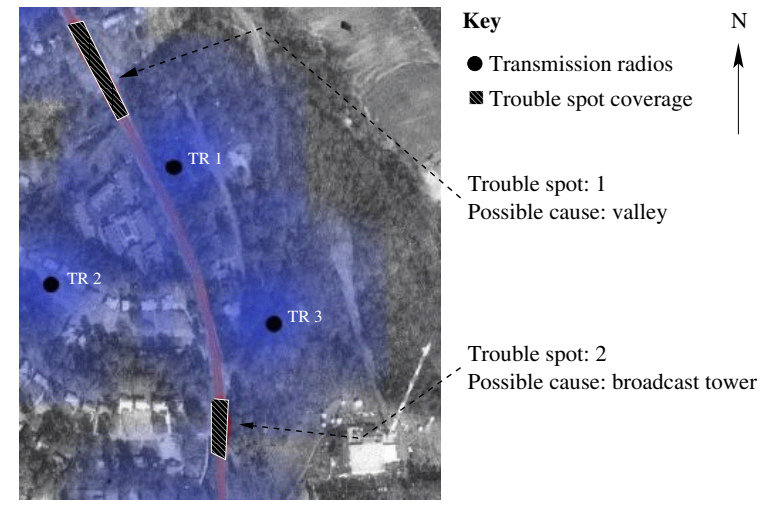

Fig. 2. A geographic view of trouble spots along a road in Atlanta. The trouble spots shown here are typical areas of non communication. When traveling at $35 \mathrm{MPH}$, they cause a sharp, relatively short drop in communication (that lasts for $\approx 2 \mathrm{~s}$ ). The stretch of road shown here is $\approx 1$ mile.

of expensive membership operations i.e. as a host enters a trouble spot, it appears to the remainder of the group as though it has failed. Subsequently, the host emerges from the trouble spot, becomes recontactable only to find that it has been excluded from the group. The only option is for the device to rejoin as a new user, which is both costly and unnecessary. Note, that the situation is exasperated because disconnections can occur for other reasons (e.g. the user deactivates the device to conserve battery power). Again, this will appear to the system as a failure when in fact, it is a calculated action. In summary, traditional failure detectors are unable to differentiate between failed hosts and uncontactable hosts when employed directly in the wireless domain.

It is noteworthy to add, that we do not consider power saving actions or temporary loss of communication due to trouble spots to be anomalous events. Moreover, we postulate that this pattern of interaction should be considered the norm. For the same reasons that Janus opts to preserve virtual synchrony semantics, it is necessary to maintain the illusion of a fail-stop model of behavior in the wired domain. However, Janus will simultaneously exhibit a more sympathetic approach to the wireless pattern of behavior.

\subsection{Synchronization at the Wireless Level}

As with all asynchronous environments, processes are free to deliver messages at arbitrary rates. In the wired domain, delivery guarantees and virtual synchrony ensure that a session remains synchronized with respect to a logical ordering of events. However, under wireless conditions where latency is orders of magnitude higher, it is quite possible that a disparity can build between users. Consider the example of Jane, Bob and Paul, who all wish to hold a collaborative computing session using wireless hand-held computers on the way to work. Both Jane and Bob are lucky, in that they experience constant uninterrupted wireless connectivity. Paul on the other hand is not so lucky and encounters a number of trouble spots on his route through the network. Although Paul's messages are not necessarily lost, his mobile device delivers messages that were seen previously by Jane and Bob. If left unchecked, this disparity can build to the point where users perceive events at different times. In this example, it could be argued that 
the application can inherently tolerate a certain level of disparity, however, if Jane, Bob and Paul had been police officers co-ordinating a public order operation, the consequences could be more pronounced. As we envisage frequent wireless disconnections, it is worthwhile to maintain an asynchronous model of communication (i.e. where devices transfer messages when contactable) since a completely synchronous approach would restrict the throughput of all wireless hosts to the rate of the slowest. However, where an explicit end-to-end delivery guarantee is required, the system is forced to wait until the mobile device acknowledges the message.

\subsection{Authentication}

Secure group communication is required in any group application that involves exchanging sensitive data. Depending on the required level of security, systems may adopt different approaches. One model of security is to screen admission to the group but to relax security once admitted. In terms of Janus, the fundamental authentication issue is in relation to the envisaged model of operation. In the wired domain, failed hosts typically have to re-authenticate when re-joining the session, which, although being expensive is mitigated by the fact that wired hosts are generally more reliable than wireless devices. However, in the wireless network, it is conceivable that an unauthorized device could pose as an authorized device which is in a trouble spot. Thus, in Janus, authentication must not only be performed on entry to the system, but each time a device returns to connectivity. However, full authentication imposes a significant overhead which, in combination with high latency and frequency of trouble spots, can cause excessive delays. Note also, that security requirements vary i.e. a single solution can not suit all applications. Thus, we propose to provide facilities for end users to integrate either standard or custom security schemes in which initial authentication is of a high level, but reconnections are less security intensive.

\section{Technical Approach}

Through initial experimentation, we believe that the Janus framework is not only feasible, but will provide a practical wireless foundation that can be leveraged into a plethora of emerging projects. Moreover, we consider the goals of the Janus project to be achievable by evolving solutions to the challenges outlined above. In this section, we present a technical overview of the Janus framework, but for brevity, we limit the discussion to the issues of arbitration and synchronization.

\subsection{Arbitration: The Janus Proxy}

Proxying is a well known distributed systems approach that has been applied in a range of applications, traditionally to increase the perceived availability of some resource. However the projects literature survey provided a level of confidence that the application of proxies in this context is an effective and novel model for addressing the issues discussed above.

The proxy we envisage is diagrammatically expressed in fig. 3. In the wired domain, the proxy executes a group communication process (referred to as the fixed process), native to the target application. Through a reliable point-to-point wireless protocol, a mobile device can contact the proxy and request admission to the group. Providing that the device is authenticated appropriately, a fixed process is instantiated in the wired domain 


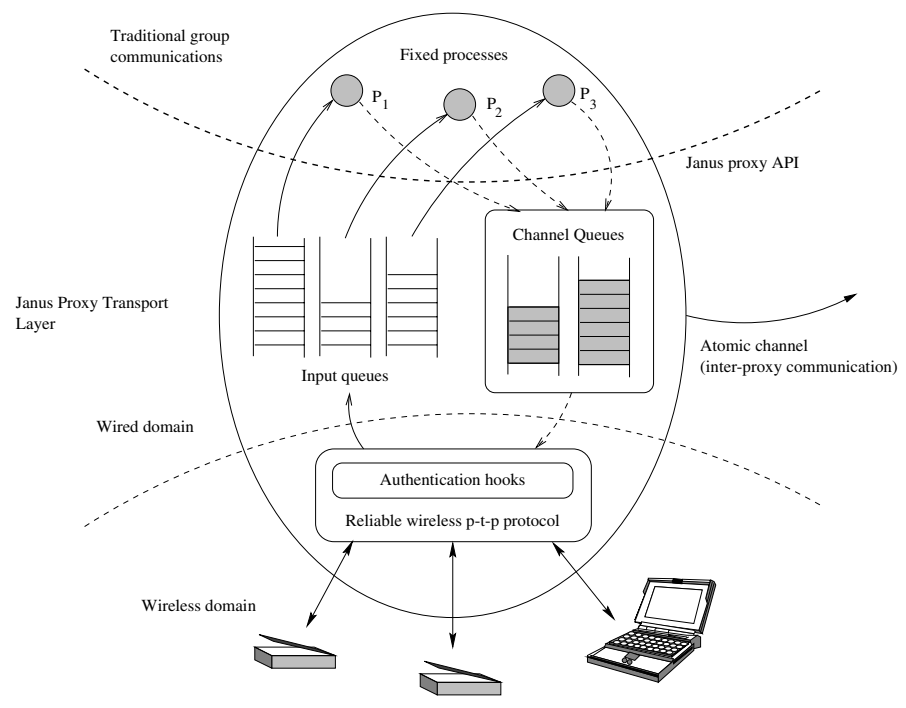

Fig. 3. An architectural view of a Janus proxy.

and the required channels are initialized. Note, that this approach offers two notable advantages, firstly, because the fixed process is viewed as any other wired participant, the semantics of the group communication system are maintained and, secondly, each mobile device becomes individually addressable. Once part of the group, the mobile device can exchange messages with the wired participants through a number of queues. On group admission, each wireless host is provided with an input queue viz. a queue of messages transmitted by the mobile device that are in turn presented to the fixed process for wired transmission in the manner native to the target application. Intuitively, one would expect a second queue to be present to pass messages from the wired domain to the mobile device. However, in multichannel group communication architectures, we suggest that it is more effective to store one queue of messages per channel and for the proxy to maintain a pointer into the list for each mobile host that is a member of the channel. It is noteworthy to add that another reason this method was adopted was to facilitate a level of synchronization at the wireless host, and as such, this discussion is expanded upon in section 5.2. Finally, when a wireless host wishes to leave the session, the corresponding fixed process is instructed to leave, the applications native leave protocol is executed and the process is terminated along with any redundant queues.

\subsection{Synchronization}

We postulate that virtual synchrony is prohibitively expensive to apply directly in the wireless domain, unless delivery ordering guarantees are specifically required. However, an element of synchronization is necessary at the wireless level to maintain consistency (at least in terms of user perception). In Janus, two levels of synchrony are supported, but for brevity, only proxy level synchronization is discussed.

Single Proxy Synchronization The Janus proxy facilitates message exchange through queues that are instantiated on a per channel basis and represent the mobile hosts 
progress with pointers. These are denoted as current $_{c}^{i}$ or $C U_{c}^{i}$ where $i$ refers to the host and $c$ is the channel. Two additional pointers store the edges of a synchronization win$d o w$ for each channel: $U E_{c}$ for the upper edge and conversely $L E_{c}$ for the lower edge. Note that we define the window size $(W S)$ in terms of the windows upper and lower edges i.e. $\forall c \in$ channels $\bullet W S_{c}=U E_{c}-L E_{c}$. This is necessary to prevent the synchronization algorithm from deadlocking. Once all hosts have acknowledged a message, both the lower edge and the upper edge pointers are incremented. Thus, messages that occur before $L E_{c}$ in the message queue are guaranteed to have been received by all mobile hosts connected to a given proxy. Mobile devices are permitted to read messages asynchronously providing that a host $i$ does not reach the upper edge of the window whilst a host $j$ is at the bottom. Thus, the lag for a particular channel queue is the difference between the host that has progressed the furthest through that queue and the host that has progressed the least (i.e. $\forall c \in$ channels $\bullet \operatorname{lag}_{c}=\max \left(C U_{c}\right)-\min \left(C U_{c}\right)$ ). The event where $\operatorname{lag}_{c}$ becomes equal to $W S_{c}$ indicates that a forced resynchronisation is necessary to maintain consistency amongst the wireless hosts. Resynchronisation is performed by initially identifying those hosts that are at the bottom of the message list and placing them in a set called slow. For each host in slow, a query is passed to the proxy in question to see if the host is in a known trouble spot or if it has just emerged from a trouble spot (and so is catching up). Those hosts for which the latter is the case, remain in slow, but all others are moved to a set called suspect. At this point, the upper edge of the window is increased to allow operational hosts to continue normal operation i.e. functional hosts are not constrained to the speed of the slowest and do not have to wait for failures to be confirmed. Thus, a fixed window size would not allow the algorithm to iterate and so the proxy would deadlock. On the next resynchronisation, hosts are again classified as slow or suspect. If the number of successive iterations in which a host has been classified as slow exceeds a threshold $t h r_{1}$, then that host is

Algorithm 1: Single Proxy Synchronization

Code for proxy $P_{i}$ with respect to an arbitrary channel $c$

Initially $W S_{c}=W S^{\prime}($ initialwindow size $), t h r_{1}=$ slowthreshold, $t h r_{2}=$ suspect threshold

1: while $\left(\operatorname{lag}_{c} \neq W S_{c}\right) / /$ Everything is nominal

2: $w_{i}$ reads a message $\Rightarrow C U_{c}^{i}=C U_{c}^{i}+1$

3: $\neg\left(\exists w_{i} \in\right.$ hosts $\bullet w_{i}$ is a member of $\left.c \wedge C U_{c}^{i}=L E_{c}\right) \Rightarrow$

4: $\left(\right.$ slow $\cup$ suspect $\left.=\emptyset \wedge W S>W S^{\prime}\right) \Rightarrow L E_{c}=L E_{c}+1 / /$ Shrink window

5: $\left(\right.$ slow $\cup$ suspect $\left.=\emptyset \wedge W S=W S^{\prime}\right) \Rightarrow U E_{c}=U E_{c}+1 \wedge L E_{c}=L E_{c}+1$

6: $\left(\operatorname{lag}_{c}=W S_{c}\right) \Rightarrow / /$ Force synchronization

7: slow $=$ slow $\backslash\left\{w_{i} \mid w_{i} \in\right.$ hosts $\wedge w_{i} \in$ slow $\left.\bullet C U_{c}^{i}=U E_{c}\right\}$

8: suspect $=$ suspect $\backslash\left\{w_{i} \mid w_{i} \in\right.$ hosts $\wedge w_{i} \in$ suspect $\left.\bullet C U_{c}^{i} \neq L E_{c}\right\} / /$ Prune normal hosts

9: slow $=$ slow $\cup\left\{w_{i} \mid w_{i} \in\right.$ hosts $\left.\bullet C U_{c}^{i}=L E_{c}^{i}\right\}$

10: $\forall w_{i} \in \operatorname{slow} \bullet \operatorname{count}\left(w_{i}\right.$, slow $)=t h r_{1} \vee \neg\left(w_{i}\right.$ is in a known trouble spot $) \Rightarrow$ slow $=$ slow $\backslash\left\{w_{i}\right\} \wedge$ suspect $=$ suspect $\cup\left\{w_{i}\right\}$

11: $\forall w_{i} \in \operatorname{suspect} \bullet \operatorname{count}\left(w_{i}\right.$, suspect $)=t h r_{2} \Rightarrow$ kill(fixed process $\left.s_{i}\right)$

12: $($ slow $\cup$ suspect $\neq \emptyset) \Rightarrow U E_{c}=U E_{c}+K / /$ Increase $U E_{c}$ by some constant $K$

Fig. 4. The informal Single Proxy Synchronization algorithm. 
added to suspect. Similarly, if the number of successive iterations in which a host has been classified as suspect exceeds a threshold $t h r_{2}$, then the host is deemed to have failed and its fixed process is killed. In the wired domain, this action is viewed as a failstop failure and is detected and handled through the applications native mechanisms; thus, the underlying semantics of the group communication system are again upheld. Based on this, we present the single proxy synchronization algorithm in fig. 4.

\section{Conclusion}

This paper has opened a discussion on the Janus architecture that is developing strong group communications for mobile wireless environments. In this context, strong group communications has been touted as the basis for a number of emerging application domains (e.g. mobile commerce). In section 4 we presented the issues that oppose this goal and subsequently an exemplar solution was proposed to tackle one of these.

Future work will continue the development of these concepts, the envisaged culmination being a usable software framework that can be easily integrated into any traditional strong group communications system.

\section{References}

1. D. A. Agarwal. Totem: A Reliable Ordered Delivery Protocol for Interconnected Local-Area Networks. PhD thesis, University of California, Santa Barbara, 1994.

2. K. Berket. The InterGroup Protocols: Scalable Group Communication for the Internet. $\mathrm{PhD}$ thesis, University of California, Santa Barbara, December 2000.

3. K. P. Birman. The Process Group Approach to Reliable Distributed Computing. Communications of The ACM, pages 37-53, December 1993.

4. K. P. Birman. Building Secure and Reliable Network Applications. Prentice Hall, 1997. Available at: http://www.cs.cornell.edu/ken/.

5. S. Deering. Host Extensions for IP Multicasting. Network Working Group, Stanford University, August 1989. Available from: http://www.faqs.org/rfcs/rfc1112.html.

6. D. Dolev and D. Malki. The Transis Approach to High Availability Cluster Communication. In Communications of the ACM, April 1996.

7. J. Dunlop and D. G. Smith. Telecommunications Engineering. Chapman and Hall, third edition, 1998.

8. P. Gray and J. S. Pascoe. On Group Communication Systems: Inisght, a Primer and a Snapshot. In Proc. of The 2001 International Conference on Computational Science, Lecture Notes in Computer Science, May 2001.

9. W. C. Y. Lee. Mobile Communications Engineering. McGraw Hill, second edition, 1997.

10. L. E. Moser, Y. Amir, P. M. Melliar-Smith, and D. A. Agarwal. Extended Virtual Synchrony. In International Conference on Distributed Computing Systems, pages 56-65, 1994.

11. L. E. Moser, P. M. Melliar-Smith, D. A. Agarwal, R. K. Budhia, and C. A. LingleyPapadopoulos. Totem: A Fault-Tolerant Multicast Group Communication System. In Communications of the ACM, April 1996.

12. J. S. Pascoe, V. S. Sunderam, U. Varshney, and R. J. Loader. Middleware Enhancements for Metropolitan Area Wireless Internet Access. Future Generation Computer Systems, Expected: 2002. In press.

13. T. Rappaport. Wireless Communications Principles and Practice. Prentice Hall, 1996.

14. O. Rodeh, K. P. Birman, and D. Dolev. The Architecture and Performance of Security Protocols in the Ensemble Group Communication System. Technical Report TR2000-1791, Cornell University, March 2000.

15. R. van Renesse, K. P. Birman, and S. Maffeis. Horus, A Flexible Group Communication System. In Communications of the ACM, April 1996. 